\title{
Applications of Hofer's geometry to Hamiltonian dynamics
}

\author{
Felix Schlenk*
}

Abstract. We prove that for every subset $A$ of a tame symplectic manifold $(W, \omega)$ meeting a semi-positivity condition, the $\pi_{1}$-sensitive Hofer-Zehnder capacity of $A$ is not greater than four times the stable displacement energy of $A$,

$$
c_{\mathrm{HZ}}^{\circ}(A, W) \leq 4 e\left(A \times S^{1}, W \times T^{*} S^{1}\right) .
$$

This estimate yields almost existence of periodic orbits near stably displaceable energy levels of time-independent Hamiltonian systems. Our main applications are:

- The Weinstein conjecture holds true for every stably displaceable hypersurface of contact type in $(W, \omega)$.

- The flow describing the motion of a charge on a closed Riemannian manifold subject to a non-vanishing magnetic field and a conservative force field has contractible periodic orbits at almost all sufficiently small energies.

The proof of the above energy-capacity inequality combines a curve shortening procedure in Hofer geometry with the following detection mechanism for periodic orbits: If the ray $\left\{\varphi_{F}^{t}\right\}$, $t \geq 0$, of Hamiltonian diffeomorphisms generated by a compactly supported time-independent Hamiltonian stops to be a minimal geodesic in its homotopy class, then a non-constant contractible periodic orbit must appear.

Mathematics Subject Classification (2000). 37J05, 37J45, 58F05.

Keywords. Hofer-Zehnder capacity, displacement energy, Weinstein conjecture, periodic orbits.

\section{Introduction and results}

On their search for periodic orbits of autonomous Hamiltonian systems, Hofer and Zehnder ([27], [28]) associated to every open subset $A$ of a symplectic manifold $(V, \omega)$ a number, the Hofer-Zehnder capacity $c_{\mathrm{HZ}}(A) \in[0, \infty]$, in such a way that $c_{\mathrm{HZ}}(A)<\infty$ implies almost existence of periodic orbits near any compact regular

\footnotetext{
${ }^{*}$ Research partially supported by the von Roll Research Foundation
} 
energy level of an autonomous Hamiltonian system on $A$. Showing that $c_{\mathrm{HZ}}(A)$ is finite is, however, often a difficult problem. Our main result is that if a subset $A$ of a tame symplectic manifold meeting a suitable semi-positivity condition can be displaced from itself by a Hamiltonian isotopy in a stabilized sense, then the HoferZehnder capacity of $A$ is indeed finite.

In order to set notations, we abbreviate $I=[0,1]$ and consider an arbitrary smooth symplectic manifold $(V, \omega)$ without boundary. Denote by $\mathscr{H}_{c}(I \times V)$ the set of smooth functions $I \times V \rightarrow \mathbb{R}$ with compact support. The Hamiltonian vector field of $H \in \mathscr{H}_{c}(I \times V)$, defined by

$$
\omega\left(X_{H_{t}}, \cdot\right)=-d H_{t}(\cdot),
$$

generates a flow $h_{t}$. The time-1-maps $h$ form the group

$$
\operatorname{Ham}_{c}(V, \omega):=\left\{h \mid H \in \mathscr{H}_{c}(I \times V)\right\}
$$

of compactly supported Hamiltonian diffeomorphisms of $(V, \omega)$. The set of functions in $\mathscr{H}_{c}(I \times V)$ which do not depend on $t \in I$ is denoted by $\mathscr{H}_{c}(V)$. We shall denote functions in $\mathscr{H}_{c}(I \times V)$ by $H$ or $K$ and functions in $\mathscr{H}_{c}(V)$ by $F$ or $G$, and their flows by $h_{t}$ or $k_{t}$ and $f_{t}$ or $g_{t}$.

The Hofer-Zehnder capacity we shall study is defined as follows. We say that $F \in \mathscr{H}_{c}(V)$ is slow if all non-constant contractible periodic orbits of $f_{t}$ have period greater than 1. Following [27], [28] and [38], [53], [17] we define for each subset $A$ of $(V, \omega)$ the $\pi_{1}$-sensitive Hofer-Zehnder capacity

$$
c_{\mathrm{HZ}}^{\circ}(A, V, \omega)=\sup \left\{\max F-\min F \mid F \in \mathscr{H}_{c}(\operatorname{Int}(A)) \text { is slow }\right\} .
$$

We shall often suppress $\omega$ from the notation, and we shall write $c_{\mathrm{HZ}}^{\circ}(V)$ instead of $c_{\mathrm{HZ}}^{\circ}(V, V)$. The Hofer-Zehnder capacity $c_{\mathrm{HZ}}(A)$ mentioned above is obtained by taking the supremum over the smaller class of functions $F \in \mathscr{H}_{c}(\operatorname{Int}(A))$ for which all non-constant periodic orbits of $f_{t}$ have period $>1$. Therefore, $c_{\mathrm{HZ}}(A) \leq c_{\mathrm{HZ}}^{\circ}(A, V)$.

We shall compare the Hofer-Zehnder capacity $c_{\mathrm{HZ}}^{\circ}(A, V)$ with the displacement energy defined in [21], [32]. The norm $\|H\|$ of $H \in \mathscr{H}_{c}(I \times V)$ is defined as

$$
\|H\|=\int_{0}^{1}\left(\max _{x \in V} H(t, x)-\min _{x \in V} H(t, x)\right) d t,
$$

and the displacement energy $e(A, V)=e(A, V, \omega) \in[0, \infty]$ of a subset $A$ of $V$ is defined as

$$
e(A, V)=\inf \left\{\|H\| \mid H \in \mathscr{H}_{c}(I \times V), h(A) \cap A=\emptyset\right\}
$$

if $A$ is compact and as

$$
e(A, V)=\sup \{e(K, V) \mid K \subset A \text { is compact }\}
$$


for a general subset $A$ of $V$. In fact, we shall compare $c_{\mathrm{HZ}}^{\circ}(A, V)$ with the stable displacement energy defined as

$$
e_{S}(A, V):=e\left(A \times S^{1}, V \times T^{*} S^{1}, \omega \oplus \omega_{0}\right)
$$

where $\omega_{0}=d p \wedge d q$ is the standard symplectic form on $T^{*} S^{1}$. We are able to do this for the following class of symplectic manifolds.

Definition ([20], [56], [2]). A symplectic manifold $(W, \omega)$ is tame if $W$ admits an almost complex structure $J$ and a complete Riemannian metric $g$ such that

- $J$ is uniformly tame, i.e., there are positive constant $C_{1}$ and $C_{2}$ such that

$$
\omega(X, J X) \geq C_{1}\|X\|^{2} \quad \text { and } \quad|\omega(X, Y)| \leq C_{2}\|X\|\|Y\|
$$

for all $X, Y \in T W$.

- The sectional curvature of $(W, g)$ is bounded from above and the injectivity radius of $(W, g)$ is bounded away from zero.

Examples of tame symplectic manifolds are closed symplectic manifolds, standard cotangent bundles $\left(T^{*} M, \omega_{0}\right)$ as well as twisted cotangent bundles $\left(T^{*} M, \omega_{\sigma}\right)$ over a closed base $M$, and symplectic manifolds which at infinity are isomorphic to the symplectization of a closed contact manifold. The class of tame symplectic manifolds is closed under taking products or coverings.

For technical reasons we also impose a semi-positivity condition on $(W, \omega)$. The first Chern class $c_{1} \in H^{2}(W ; \mathbb{Z})$ is defined as the first Chern class of the complex vector bundle $(T W, J)$, where $J$ is any almost complex structure such that $\omega(\cdot, J \cdot)$ is a Riemannian metric. Recall from [43], [23], [54], [44] that a $2 n$-dimensional symplectic manifold $(W, \omega)$ is strongly semi-positive if for all $A \in \pi_{2}(W)$,

$$
\omega(A)>0, c_{1}(A) \geq 2-n \Longrightarrow c_{1}(A) \geq 0 .
$$

Definition. A $2 n$-dimensional symplectic manifold $(W, \omega)$ is stably strongly semipositive if for all $A \in \pi_{2}(W)$,

$$
\omega(A)>0, c_{1}(A) \geq 1-n \Longrightarrow c_{1}(A) \geq 0 .
$$

Equivalently, $(W, \omega)$ satisfies one of the following conditions.

(i) $\omega(A)=\lambda c_{1}(A)$ for every $A \in \pi_{2}(W)$ and some $\lambda \geq 0$;

(ii) $c_{1}(A)=0$ for every $A \in \pi_{2}(W)$;

(iii) The minimal Chern number $N \geq 0$ defined by $c_{1}\left(\pi_{2}(W)\right)=N \mathbb{Z}$ is at least $n$. 
Since $\left(T^{*} S^{1}, \omega_{0}\right)$ is exact and has vanishing first Chern class, $(W, \omega)$ is stably strongly semi-positive if and only if $\left(W \times T^{*} S^{1}, \omega \oplus \omega_{0}\right)$ is strongly semi-positive. This assumption guarantees that the evaluation map used in the definition of the Gromov-Witten invariants relevant for our arguments is a pseudo-cycle. If one is willing to use Liu-Tian's construction of the $S^{1}$-invariant virtual moduli cycle, this assumption can be dropped throughout the paper.

Our main result is the following energy-capacity inequality.

Theorem 1.1. Assume that $A$ is a subset of a tame and stably strongly semi-positive symplectic manifold $(W, \omega)$. Then

$$
c_{\mathrm{HZ}}^{\circ}(A, W) \leq 4 e_{S}(A, W) .
$$

We shall derive Theorem 1.1 from the following result by capitalizing on the fact that the definition of $c_{\mathrm{HZ}}^{\circ}$ involves only contractible periodic orbits and by using a stabilization trick found in Macarini's work [41].

Theorem 1.2. Assume that $A$ is a subset of a tame and strongly semi-positive symplectic manifold $(W, \omega)$. Then

$$
c_{\mathrm{HZ}}^{\circ}(A, W) \leq 4 e(A, W) .
$$

Up to its slightly more restrictive hypothesis, Theorem 1.1 is stronger than Theorem 1.2. Indeed, it is elementary to see that $e_{S}(A, V) \leq e(A, V)$ in general, and in the dynamically relevant Example 1.5 below we have $e_{S}(A, V)<e(A, V)=\infty$.

The energy-capacity inequality

$$
c_{\mathrm{HZ}}^{\circ}(A, V) \leq e(A, V)
$$

is known for every subset $A$ of a weakly exact symplectic manifold $(V, \omega)$ which is closed or convex ([22], [53], [12], [16], [11]). For the open ball $B^{2 n}(r)$ of radius $r$ in $\left(\mathbb{R}^{2 n}, \omega_{0}\right)$ it holds that

$$
c_{\mathrm{HZ}}^{\circ}\left(B^{2 n}(r), \mathbb{R}^{2 n}\right)=e\left(B^{2 n}(r), \mathbb{R}^{2 n}\right)=\pi r^{2},
$$

see [28], and so (2) is sharp. It is conceivable that the factor 4 in Theorems 1.1 and 1.2 can be omitted

Following Polterovich [50] we shall obtain Theorem 1.2 by combining an elementary curve shortening technique in Hofer's geometry with the following detection mechanism for periodic orbits.

Theorem 1.3. Assume that $(W, \omega)$ is a tame and strongly semi-positive symplectic manifold, and that the autonomous Hamiltonian $F \in \mathscr{H}_{c}(W)$ is slow. Then the path $f_{t}, t \in[0,1]$, is length minimizing in its homotopy class. 
Here, the length of $f_{t}$ is defined as $\|F\|$. This result was discovered by Hofer [22] for $\left(\mathbb{R}^{2 n}, \omega_{0}\right)$ and has been proved in [34] for weakly exact tame symplectic manifolds; it removes an additional assumption on $F$ in [9], [44] and verifies Conjecture 1.2 in [44] for tame strongly semi-positive symplectic manifolds.

Theorems 1.1 and 1.2 show that if $e_{S}(A, W)$ or $e(A, W)$ is finite, then so is $c_{\mathrm{HZ}}^{\circ}(A, W)$, and the finiteness of $c_{\mathrm{HZ}}^{\circ}(A, W)$ implies existence of contractible periodic orbits on almost every compact regular energy level of an autonomous Hamiltonian system on $A$. We thus want to understand which compact subsets of a symplectic manifold $V$ have finite (stable) displacement energy. Every compact subset of a symplectic manifold of the form $\left(V \times \mathbb{R}^{2}, \omega \oplus \omega_{0}\right)$ has finite displacement energy. Less obvious sufficient assumptions on $A$ alone are collected in the following proposition essentially due to Laudenbach [35] and to Polterovich [49] and Laudenbach-Sikorav [36]. Recall that a middle-dimensional submanifold $L$ of a symplectic manifold $(V, \omega)$ is called Lagrangian if $\omega$ vanishes on $L$.

Proposition 1.4. Let $A$ be a compact subset of a $2 n$-dimensional symplectic manifold $(V, \omega)$.

(i) If $A$ is contained in an embedded finite $C W$-complex $X$ of dimension $<n$, then $e_{S}(A, V)<\infty$.

(ii) If $A$ is contained in an n-dimensional closed submanifold $M$ which is not Lagrangian, then $e_{S}(A, V)=0$.

(iii) If $A$ is strictly contained in a closed Lagrangian submanifold $L$, then $e_{S}(A, V)=0$.

The example $S^{1} \subset\left(T^{*} S^{1}, \omega_{0}\right)$ shows that neither the dimension assumption in (i) nor the assumption $\left.\omega\right|_{M} \neq 0$ in (ii) nor the assumption $A \subsetneq L$ in (iii) can be omitted. The following example will play an important role in our applications.

Example 1.5. Let $\sigma$ be a non-vanishing closed 2-form on a closed manifold $M$ and let $\omega_{\sigma}=\omega_{0}+\pi^{*} \sigma$ be the twisted symplectic form on its cotangent bundle $\pi: T^{*} M \rightarrow M$. Then $e_{S}\left(M, T^{*} M, \omega_{\sigma}\right)=0$ by Proposition 1.4 (ii). Note that if the Euler characteristic $\chi(M)$ does not vanish, then $e\left(M, T^{*} M, \omega_{\sigma}\right)=\infty$.

Theorems 1.1 and 1.2 and Proposition 1.4, which are proved in the next section, have various applications to the existence problem of periodic orbits of timeindependent Hamiltonian systems. Some of them are given in Section 3 below. Further such applications as well as an application to Lagrangian intersections can be found in [52].

Acknowledgements. The cornerstone to this work was laid by Leonid Polterovich, who suggested to me to combine his approach to periodic orbits of a charge in a 
magnetic field in [50] with the approach in [12]. I cordially thank him for sharing his insight with me. I also thank Urs Frauenfelder and Viktor Ginzburg for their generous help, and Ely Kerman and Jean-Claude Sikorav for valuable discussions. Much of this work has been written during my stay at Tel Aviv University in April 2003, and it was finished at FIM of ETH Zürich and at Leipzig University. I wish to thank these institutions for their support, and I thank Hari and Harald and Matthias Schwarz for their warm hospitality.

\section{Proofs}

2.1. Proof of Theorem 1.2. We follow Polterovich's beautiful argument in [50, Section 9.A]. The proof consists of two steps.

\section{Step 1. Curve shortening in Hofer's geometry}

Curve shortening in Hofer's geometry was invented by Sikorav in [55] and further developed in [33, Proposition 2.2]. Here, we closely follow the proof of Theorem 8.3.A in [51], see also Theorem 3.3.A in [3].

We consider an arbitrary symplectic manifold $(V, \omega)$. Two Hamiltonians $H, K \in$ $\mathscr{H}_{c}(I \times V)$ are equivalent, $H \sim K$, if $h=k$ and the paths $\left\{h_{t}\right\},\left\{k_{t}\right\}, t \in[0,1]$, are homotopic in $\operatorname{Ham}_{c}(V, \omega)$ with fixed end points. In other words, there exists a smooth family $\left\{H^{s}\right\}, s \in[0,1]$, in $\mathscr{H}_{c}(I \times V)$ such that $h_{t}^{0}=h_{t}$ and $h_{t}^{1}=k_{t}$ for all $t$ and $h^{s}=h=k$ for all $s$. The group of equivalence classes $\mathscr{H}_{c}(I \times V) / \sim$ form the universal cover $\widetilde{\operatorname{Ham}}_{c}(V, \omega)$ of $\operatorname{Ham}_{c}(V, \omega)$. We denote the lift of the Hofer norm to $\widetilde{\operatorname{Ham}}_{c}(V, \omega)$ by

$$
\rho\left[h_{t}\right] \equiv \rho[H]:=\inf \{\|K\| \mid K \sim H\} .
$$

Proposition 2.1. Consider a compact subset $A$ of an arbitrary symplectic manifold $(V, \omega)$ such that $e(A, V)<\infty$. If $F: V \rightarrow \mathbb{R}$ is supported in $A$ and $\|F\|>4 e(A, V)$, then $\rho[F]<\|F\|$.

Proof. Choose a path $\left\{h_{t}\right\}, t \in[0,1]$, in $\operatorname{Ham}_{c}(V, \omega)$ such that $h(A) \cap A=\emptyset$ and

$$
\rho\left[h_{t}\right]<\frac{1}{4}\|F\| .
$$

For $t \in[0,1]$ we decompose the path $f_{t}$ as

$$
f_{t}=\left(f_{t / 2} \circ h_{t} \circ f_{t / 2} \circ h_{t}^{-1}\right) \circ\left(h_{t} \circ f_{t / 2}^{-1} \circ h_{t}^{-1} \circ f_{t / 2}\right) \equiv b_{t} \circ a_{t} .
$$

As we shall see below,

$$
\rho\left[a_{t}\right]<\frac{1}{2}\|F\| \quad \text { and } \quad \rho\left[b_{t}\right] \leq \frac{1}{2}\|F\| .
$$


Since $\left\{b_{t} \circ a_{t}\right\}$ is equivalent to the juxtaposition of $\left\{a_{t}\right\}$ and $\left\{b_{t} \circ a_{1}\right\}$ and since $\rho$ satisfies the triangle inequality, the estimates (4) imply Proposition 2.1. In order to prove the first estimate in (4), notice that the paths $\left\{f_{t / 2}^{-1} \circ h_{t}^{-1} \circ f_{t / 2}\right\}$ and $\left\{f_{1 / 2}^{-1} \circ h_{t}^{-1} \circ f_{1 / 2}\right\}$ are equivalent and that

$$
\rho\left[f_{1 / 2}^{-1} \circ h_{t}^{-1} \circ f_{1 / 2}\right]=\rho\left[h_{t}^{-1}\right]=\rho\left[h_{t}\right] .
$$

Together with the triangle inequality and the estimate (3) we can estimate

$$
\begin{aligned}
\rho\left[a_{t}\right] & =\rho\left[h_{t} \circ f_{t / 2}^{-1} \circ h_{t}^{-1} \circ f_{t / 2}\right] \\
& \leq \rho\left[h_{t}\right]+\rho\left[f_{t / 2}^{-1} \circ h_{t}^{-1} \circ f_{t / 2}\right] \\
& =2 \rho\left[h_{t}\right] \\
& <\frac{1}{2}\|F\| .
\end{aligned}
$$

To prove the second estimate in (4), notice that the path $\left\{b_{t}\right\}=\left\{f_{t / 2} \circ h_{t} \circ f_{t / 2} \circ h_{t}^{-1}\right\}$ is equivalent to the path $\left\{f_{t / 2} \circ h \circ f_{t / 2} \circ h^{-1}\right\}$ generated by the Hamiltonian

$$
K(t, x)=\frac{1}{2} F(x)+\frac{1}{2} F\left(h^{-1} f_{t / 2}^{-1} x\right), \quad t \in[0,1] .
$$

Since $F$ is autonomous, $F=F \circ f_{t / 2}$, and since $h$ displaces supp $F \subset A$, so does $h^{-1}$. Therefore,

$$
\begin{aligned}
\left\|K_{t}\right\| & =\frac{1}{2}\left\|F+F \circ h^{-1} \circ f_{t / 2}^{-1}\right\| \\
& =\frac{1}{2}\left\|F \circ f_{t / 2}+F \circ h^{-1}\right\| \\
& =\frac{1}{2}\left\|F+F \circ h^{-1}\right\| \\
& =\frac{1}{2}\|F\|,
\end{aligned}
$$

and so $\rho\left[b_{t}\right] \leq \frac{1}{2}\|F\|$. The proof of Proposition 2.1 is complete.

\section{Step 2. The cut point has a non-constant contractible periodic orbit}

Consider an arbitrary symplectic manifold $(V, \omega)$. We recall from the introduction that $F \in \mathscr{H}_{c}(V)$ is slow if all non-constant contractible periodic orbits of $f_{t}$ have period $>1$. We say that $F \in \mathscr{H}_{c}(V)$ is flat if all non-constant periodic orbits of the linearized flow of $F$ at its critical points have period $>1$.

Lemma 2.2. Assume that $(W, \omega)$ is a tame strongly semi-positive symplectic manifold, and that the autonomous Hamiltonian $F \in \mathscr{H}_{c}(W)$ is slow and flat. Then the path $f_{t}, t \in[0,1]$, is length minimizing in its homotopy class.

Proof. If $W$ is closed, this result is proved in [9], [44], see also [34]. If $(W, \omega)$ is not closed but tame, then the compactness theorems in [20], [56] hold, and so the arguments in [44] establishing compactness of the relevant Floer moduli space go through. 
Following a suggestion by Viktor Ginzburg, we derive Theorem 1.3 from Lemma 2.2 by elementary means:

Proof of Theorem 1.3. Let $F \in \mathscr{H}_{c}(W)$ be slow. Arguing by contradiction, we assume that $\rho[F]<\|F\|$. Choose $\varepsilon>0$ so small that

$$
\rho[F]+2 \varepsilon<\|F\| .
$$

Since $F$ is smooth and compactly supported and by Sard's theorem, the set $C$ of critical values of $F$ is compact and has zero Lebesgue measure. If $F(W)=[a, b]$, we thus find finitely many intervals $\left[a_{i}, b_{i}\right] \subset[a, b] \backslash C$ such that $\sum_{i}\left(b_{i}-a_{i}\right) \geq$ $(b-a)-\varepsilon$. Choose a smooth function $r:[a, b] \rightarrow \mathbb{R}$ such that $r(a)=a$ and such that $0 \leq r^{\prime}(t) \leq 1$ for all $t$ and

$$
r^{\prime}(t)=1 \text { if } t \in \bigcup_{i}\left[a_{i}, b_{i}\right] \quad \text { and } \quad r^{\prime}(t)=0 \text { if } t \in C .
$$

The function $G=r \circ F$ belongs to $\mathscr{H}_{c}(W)$ and is both slow and flat. Moreover,

$$
\max G=r(b) \geq r(a)+(b-a)-\varepsilon=\max F-\varepsilon .
$$

Since the path $\left\{g_{t} \circ f_{t}^{-1}\right\}$ is generated by $G-F=r \circ F-F$ and since $\|r \circ F-F\|=$ $\max F-\max G \leq \varepsilon$, we have $\rho\left[g_{t} \circ f_{t}^{-1}\right] \leq \varepsilon$. Therefore,

$$
\begin{aligned}
\rho[G] & =\rho\left[g_{t} \circ f_{t}^{-1} \circ f_{t}\right] \\
& \leq \rho\left[g_{t} \circ f_{t}^{-1}\right]+\rho[F] \\
& \leq \varepsilon+\rho[F] \\
& <\|F\|-\varepsilon \\
& \leq\|G\| .
\end{aligned}
$$

We have constructed a slow and flat $G \in \mathscr{H}_{c}(W)$ with $\rho[G]<\|G\|$, in contradiction to Lemma 2.2.

We would like to point out that the proof of Lemma 2.2 is the only place were we use a semi-positivity assumption on $(W, \omega)$. As explained in [44] the $S^{1}$-invariant virtual moduli cycle can be used to establish Lemma 2.2 for arbitrary tame symplectic manifolds. The above argument then yields Theorem 1.3 and hence Conjecture 1.2 in [44] for all tame symplectic manifolds.

End of the proof of Theorem 1.2. We can assume that $e(A, W)<\infty$, and in view of the definitions of the capacity $c_{\mathrm{HZ}}^{\circ}$ and the displacement energy $e$ we can assume that $A$ is compact. Let $F \in \mathscr{H}_{c}$ (Int $A$ ) be such that $\max F-\min F=\|F\|>4 e(A, W)$. According to Proposition 2.1 we have $\rho[F]<\|F\|$, and so Theorem 1.3 shows that $F$ is not slow. Therefore, $c_{\mathrm{HZ}}^{\circ}(A, W) \leq 4 e(A, W)$. 
2.2. Proof of Theorem 1.1. We shall derive Theorem 1.1 from Theorem 1.2 by a stabilization argument. Let $G(q, p)=\frac{1}{2} p^{2}$ be the Hamiltonian generating the geodesic flow on $T^{*} S^{1}$, and abbreviate $G^{\varepsilon}=\{(q, p) \mid G(q, p) \leq \varepsilon\}$.

Lemma 2.3. For any subset $A$ of a symplectic manifold $(V, \omega)$ and any $\varepsilon>0$,

$$
c_{\mathrm{HZ}}^{\circ}(A, V) \leq c_{\mathrm{HZ}}^{\circ}\left(A \times G^{\varepsilon}, V \times T^{*} S^{1}\right) .
$$

Proof. We can assume that Int $A \neq \emptyset$. Let $F \in \mathscr{H}_{c}$ (Int $A$ ) be slow. We choose a smooth function $a: \mathbb{R} \rightarrow[0,1]$ such that

$$
a(t)=1 \text { if } t \leq \frac{1}{3} \varepsilon \quad \text { and } \quad a(t)=0 \text { if } t \geq \frac{2}{3} \varepsilon .
$$

The function $F_{S}: V \times T^{*} S^{1} \rightarrow \mathbb{R}$ given by $(v, w) \mapsto F(v) a(G(w))$ belongs to $\mathscr{H}_{c}\left(\right.$ Int $\left.\left(A \times G^{\varepsilon}\right)\right)$. In order to see that $F_{S}$ is slow, assume that $x(t)$ is a contractible periodic orbit of its Hamiltonian flow. Then $x(t)=\left(x_{1}(t), x_{2}(t)\right) \subset V \times T^{*} S^{1}$, where both $x_{1}(t)$ and $x_{2}(t)$ are contractible periodic orbits. Denoting the Hamiltonian vector fields of $F$ and $G$ by $X_{F}$ and $X_{G}$, we find

$$
\begin{aligned}
& \dot{x}_{1}(t)=a\left(G\left(x_{2}(t)\right)\right) X_{F}\left(x_{1}(t)\right), \\
& \dot{x}_{2}(t)=F\left(x_{1}(t)\right) a^{\prime}\left(G\left(x_{2}(t)\right)\right) X_{G}\left(x_{2}(t)\right) .
\end{aligned}
$$

Therefore, the orbits $x_{1}(t)$ and $x_{2}(t)$ are, up to reparametrization, orbits of $X_{F}$ and $X_{G}$. Since $F$ and $G$ are autonomous, we conclude that the functions $a\left(G\left(x_{2}(t)\right)\right)$ and $F\left(x_{1}(t)\right) a^{\prime}\left(G\left(x_{2}(t)\right)\right)$ are constant. Since $\left|a\left(G\left(x_{2}\right)\right)\right| \in[0,1]$ and $F$ is slow, the orbit $x_{1}(t)$ is constant or has period $>1$, and since all contractible periodic orbits of the flow of $G$ are constant, the orbit $x_{2}(t)$ is constant. We have constructed for every slow $F \in \mathscr{H}_{c}(\operatorname{Int} A)$ a slow $F_{S} \in \mathscr{H}_{c}\left(\operatorname{Int}\left(A \times G^{\varepsilon}\right)\right)$ with $\max F=\max F_{S}$. Lemma 2.3 thus follows.

In order to prove Theorem 1.1 we need to show that for every compact subset $A$ of $W$,

$$
c_{\mathrm{HZ}}^{\circ}(A, W) \leq 4 e\left(A \times S^{1}, W \times T^{*} S^{1}\right) .
$$

We can assume that $e\left(A \times S^{1}, W \times T^{*} S^{1}\right)$ is finite. Fix $\delta>0$, and choose $H \in$ $\mathscr{H}_{c}\left(I \times W \times T^{*} S^{1}\right)$ such that $h$ displaces $A \times S^{1}$ and

$$
\|H\| \leq e\left(A \times S^{1}, W \times T^{*} S^{1}\right)+\delta .
$$

We then find $\varepsilon>0$ such that $h$ displaces $A \times G^{\varepsilon}$. It follows that

$$
e\left(A \times G^{\varepsilon}, W \times T^{*} S^{1}\right) \leq\|H\| \leq e\left(A \times S^{1}, W \times T^{*} S^{1}\right)+\delta .
$$


Since both $(W, \omega)$ and $\left(T^{*} S^{1}, \omega_{0}\right)$ are tame, so is their product, and since $(W, \omega)$ is stably strongly semi-positive, $\left(W \times T^{*} S^{1}, \omega \oplus \omega_{0}\right)$ is strongly semi-positive. Together with Lemma 2.3 and Theorem 1.2 we can thus estimate

$$
\begin{aligned}
c_{\mathrm{HZ}}^{\circ}(A, W) & \leq c_{\mathrm{HZ}}^{\circ}\left(A \times G^{\varepsilon}, W \times T^{*} S^{1}\right) \\
& \leq 4 e\left(A \times G^{\varepsilon}, W \times T^{*} S^{1}\right) \\
& \leq 4 e\left(A \times S^{1}, W \times T^{*} S^{1}\right)+4 \delta .
\end{aligned}
$$

Since $\delta>0$ was arbitrary, inequality (5) follows, and so Theorem 1.1 is proved.

2.3. Proof of Proposition 1.4. (i) By assumption, the set $A \times S^{1}$ is contained in the finite $\mathrm{CW}$-complex $X \times S^{1}$ of dimension $<n+1$ in the $(2 n+2)$-dimensional symplectic manifold $\left(V \times T^{*} S^{1}, \omega \oplus \omega_{0}\right)$. Since $X \times S^{1}$ can be displaced from itself in $V \times T^{*} S^{1}$ by a smooth isotopy, a result of Laudenbach [35] implies that $X \times S^{1}$ can be displaced from itself in $\left(V \times T^{*} S^{1}, \omega \oplus \omega_{0}\right)$ by a Hamiltonian isotopy. It follows that $e_{S}(A, V) \leq e_{S}(X, V)<\infty$.

(ii) Consider the closed submanifold $M \times S^{1}$ of $V \times T^{*} S^{1}$. Since $\left.\omega\right|_{M} \neq 0$ we have $\left.\omega \oplus \omega_{0}\right|_{M \times S^{1}} \neq 0$. Moreover, the Euler characteristic of $M \times S^{1}$ vanishes. A result of Polterovich [49] and Laudenbach-Sikorav [36] thus implies that $e\left(M \times S^{1}, V \times T^{*} S^{1}\right)=0$, and so $e_{S}(A, V)=0$.

(iii) The proof of the case $n=1$ is elementary and omitted. So assume that $n \geq 2$. Since $A$ is compact, $L \backslash A$ is open. Using the Lagrangian Neighbourhood Theorem we easily find a closed submanifold $L^{\prime}$ of $V$ which is not Lagrangian and such that $A \subset L^{\prime}$. By assertion (ii) we have $e_{S}\left(L^{\prime}, V\right)=0$, and so $e_{S}(A, V)=0$.

\section{Applications}

Throughout this section, $(V, \omega)$ denotes an arbitrary symplectic manifold, while $(W, \omega)$ denotes a tame and stably strongly semi-positive symplectic manifold. We say that a compact subset $A$ of $(V, \omega)$ is displaceable if there exists $h \in \operatorname{Ham}_{c}(V, \omega)$ such that $h(A) \cap A=\emptyset$, and we say that $A$ is stably displaceable if $A \times S^{1}$ is displaceable in $\left(V \times T^{*} S^{1}, \omega \oplus \omega_{0}\right)$. Thus $A \subset V$ is (stably) displaceable if and only if $e(A, V)<\infty$ (resp. $\left.e_{S}(A, V)<\infty\right)$. Note that if $A$ is (stably) displaceable, then an entire neighbourhood of $A$ is (stably) displaceable.

\subsection{Almost existence of closed characteristics and the Weinstein conjecture.} A hypersurface $S$ in a symplectic manifold $(V, \omega)$ is a smooth compact connected orientable codimension 1 submanifold of $V$ without boundary. A closed characteristic on $S$ is an embedded circle in $S$ all of whose tangent lines belong to the distinguished 
line bundle

$$
\mathcal{L}_{S}=\left\{(x, \xi) \in T S \mid \omega(\xi, \eta)=0 \text { for all } \eta \in T_{x} S\right\}
$$

Examples show that $\mathcal{L}_{S}$ might not carry any closed characteristic, see [15], [17]. We therefore follow [26] and consider parametrized neighbourhoods of $S$. Since $S$ is orientable, there exists an open neighbourhood $I$ of 0 and a smooth diffeomorphism

$$
\vartheta: S \times I \rightarrow U \subset V
$$

such that $\vartheta(x, 0)=x$ for $x \in S$. We call $\vartheta$ a thickening of $S$, and we abbreviate $S_{\varepsilon}=\vartheta(S \times\{\varepsilon\})$. Denote by $\mathcal{P}^{\circ}\left(S_{\varepsilon}\right)$ the set of closed characteristics on $S_{\varepsilon}$ which are contractible in $V$. The refinement of the Hofer-Zehnder argument [28, Sections 4.1 and 4.2] in [42] shows

Proposition 3.1. For any thickening $\vartheta: S \times I \rightarrow U \subset V$ of a hypersurface $S$ in $(V, \omega)$ with $c_{\mathrm{HZ}}^{\circ}(U, V)<\infty$ it holds that $\mathcal{P}^{\circ}\left(S_{\varepsilon}\right) \neq \emptyset$ for almost all $\varepsilon \in I$.

Together with Theorem 1.2 we obtain

Corollary 3.2. Assume that $S$ is a stably displaceable hypersurface in $(W, \omega)$. Then for any stably displaceable thickening $\vartheta: S \times I \rightarrow U \subset W$ it holds that $\mathcal{P}^{\circ}\left(S_{\varepsilon}\right) \neq \emptyset$ for almost all $\varepsilon \in I$.

In [61], Zehnder constructed a symplectic form on the 4-torus $T^{4}=(\mathbb{R} / \mathbb{Z})^{4}$ such that none of the hypersurfaces $\left\{x_{4}=\right.$ const $\}$ carries a closed characteristic. The assumption in Corollary 3.2 that $S$ is stably displaceable thus cannot be omitted.

A hypersurface $S$ in a symplectic manifold $(V, \omega)$ is called of contact type if there exists a Liouville vector field $X$ (i.e., $\mathcal{L}_{X} \omega=d \iota_{X} \omega=\omega$ ) which is defined in a neighbourhood of $S$ and is everywhere transverse to $S$. Weinstein conjectured in [60] that every hypersurface $S$ of contact type with $H^{1}(S ; \mathbb{R})=0$ carries a closed characteristic.

Corollary 3.3. Assume that $S$ is a stably displaceable hypersurface of contact type in $(W, \omega)$. Then $\mathcal{P}^{\circ}(S) \neq \emptyset$. In particular, the Weinstein conjecture holds true for $S$.

The Weinstein conjecture has been proved for various classes of hypersurfaces of contact type in various classes of symplectic manifolds ([57], [26], [24], [10], [25], [29], [40], [58], [38], [59], [4], [37], [39], [46]). Corollary 3.3 generalizes or complements the results in [57], [26], [10], [59], [37], where the ambient symplectic manifold is of the form $\left(V \times \mathbb{R}^{2}, \omega \oplus \omega_{0}\right)$. Under the additional assumption that $(W, \omega)$ is weakly exact and convex, Corollary 3.3 has been proved in [12]. 
3.2. Periodic orbits of autonomous Hamiltonian systems. We consider a smooth proper Hamiltonian $F$ on $(V, \omega)$ which attains its minimum at 0 . We abbreviate the sublevel set $F^{-1}([0, r])$ by $F^{r}$, and define $d_{1}(F) \in[0, \infty]$ by

$$
d_{1}(F)=\sup \left\{r \in \mathbb{R} \mid F^{r} \text { is stably displaceable }\right\} .
$$

Thus $d_{1}(F)>0$ if and only if $F^{-1}(0)$ is stably displaceable. Denote by $\mathcal{P}^{\circ}\left(F^{-1}(r)\right)$ the set of non-constant periodic orbits on $F^{-1}(r)$ which are contractible in $V$. Since the set of critical values of $F$ is closed and, by Sard's theorem, of Lebesgue measure zero, Corollary 3.2 yields

Corollary 3.4. Consider a proper Hamiltonian $F$ on $(W, \omega)$ with minimum 0 , and assume that $d_{1}(F)>0$. Then $\mathcal{P}^{\circ}\left(F^{-1}(r)\right) \neq \varnothing$ for almost all $\left.\left.r \in\right] 0, d_{1}(F)\right]$.

Discussion. 1. Recall that Corollary 3.4 becomes relevant in conjunction with Proposition 1.4 applied to $A=F^{-1}(0)$.

2. According to [17], every symplectic manifold $(V, \omega)$ of dimension $2 n \geq 4$ admits a proper $C^{2}$-smooth Hamiltonian $F$ with minimum 0 and $d_{1}(F)>0$ such that for a sequence $r_{k} \rightarrow 0$ of regular values the levels $F^{-1}\left(r_{k}\right)$ carry no periodic orbit, and if $2 n \geq 6$, then $F$ can be chosen $C^{\infty}$-smooth.

3. Consider a tame symplectic manifold $\left(W^{2 n}, \omega\right)$ for which $[\omega]$ and $c_{1}$ vanish on $\pi_{2}(W)$, and assume that the proper function $F: W \rightarrow \mathbb{R}$ attains its minimum 0 along a closed symplectic submanifold $M^{2 k}$ of $(W, \omega)$. It has been shown in [17, Corollary 2.16] that $\mathcal{P}^{\circ}\left(F^{-1}(r)\right) \neq \emptyset$ for almost all $\left.\left.r \in\right] 0, b(F)\right]$, where

$$
\left.\left.b(F)=\sup \left\{r \in \mathbb{R} \mid F^{r} \subset B(M, F)\right\} \in\right] 0, \infty\right]
$$

and $B(M, F)$ is "the $F$-maximal symplectic ball neighbourhood of $M$ in $(W, \omega)$ ", see [17, Section 4.1] for details. For $k \in\{0,1, \ldots,\lfloor n / 2\rfloor\}$, this result is covered by Proposition 1.4 and Corollary 3.4 with $d_{1}(F)>0$ instead of $b(F)$. It would be interesting to compare these two constants.

3.3. Closed trajectories of a charge in a magnetic field and a potential. Consider a closed Riemannian manifold $(M, g)$ of dimension at least 2 , and endow the cotangent bundle $T^{*} M$ with the standard symplectic form $\omega_{0}=\sum_{i} d p_{i} \wedge d q_{i}$. We fix a closed 2-form $\sigma$ on $M$ and define the twisted symplectic form $\omega_{\sigma}$ on $\pi: T^{*} M \rightarrow M$ by $\omega_{\sigma}=\omega_{0}+\pi^{*} \sigma$. We also fix a function $V$ on $M$ with minimum 0 . The flow of the Hamiltonian system

$$
F_{V}:\left(T^{*} M, \omega_{\sigma}\right) \rightarrow \mathbb{R}, \quad F_{V}(q, p) \mapsto \frac{1}{2}|p|^{2}+V(q),
$$

describes (for example) the motion of a unit charge on $(M, g)$ subject to the magnetic field $\sigma$ and the potential $V$, cf. [45], [31], [14]. As before we denote by $\mathcal{P}^{\circ}\left(F_{V}^{-1}(r)\right)$ the set of periodic orbits on the level $F_{V}^{-1}(r)$ which are contractible in $T^{*} M$ and hence project to contractible closed trajectories on $M$. 
Corollary 3.5. Consider a closed Riemannian manifold $(M, g)$ endowed with a closed 2-form $\sigma$ which does not vanish identically, and let $V$ be a potential on $M$ with minimum 0 . Then $d_{1}\left(F_{V}\right)>0$ and $\mathscr{P}^{\circ}\left(F_{V}^{-1}(r)\right) \neq \emptyset$ for almost all $\left.\left.r \in\right] 0, d_{1}\left(F_{V}\right)\right]$.

Proof. It is shown in [5] that for any closed 2-form $\sigma$ on a closed manifold $M$ the symplectic manifold $\left(T^{*} M, \omega_{\sigma}\right)$ is tame. Since the kernel of the differential of the projection $\pi: T^{*} M \rightarrow M$ defines a Lagrangian distribution in the tangent bundle of $\left(T^{*} M, \omega_{\sigma}\right)$, the first Chern class vanishes, so that $\left(T^{*} M, \omega_{\sigma}\right)$ is stably strongly semi-positive. Moreover, $F_{V}$ is proper, has minimum 0 , and $F_{V}^{-1}(0) \subset M$; and since $\sigma$ does not vanish, $M$ is not Lagrangian. Proposition 1.4 (ii) thus yields $d_{1}\left(F_{V}\right)>0$, and so Corollary 3.5 follows from Corollary 3.4.

Specializing to the case $V=0$, we set $d_{1}(g, \sigma)=d_{1}\left(F_{0}\right)$ and denote the sphere bundle $F_{0}^{-1}(r)$ by $E_{r}$.

Corollary 3.6. Consider a closed Riemannian manifold $(M, g)$ endowed with a closed 2-form $\sigma$ which does not vanish identically. Then $d_{1}(g, \sigma)>0$ and $\mathcal{P}^{\circ}\left(E_{r}\right) \neq \emptyset$ for almost all $\left.\left.r \in\right] 0, d_{1}(g, \sigma)\right]$.

Discussion. 1. There has been much recent progress in the existence problem for periodic orbits of a charge in a magnetic field ([45], [31], [1], [13], [24], [14], [38], [50], [18], [30], [7], [19], [5], [17], [41], [8], [6], [12], [47]). Corollary 3.6 solves the almost existence problem at small energies. Under additional assumptions on $M, g$ or $\sigma$, stronger results are known. We refer to [14], [52], [47] for the state of the art.

2. If $\sigma$ is exact, $d_{1}(g, \sigma) \leq \frac{1}{2} \max _{x \in M}|\alpha(x)|^{2}$ for all $\alpha$ with $d \alpha=\sigma$, see [12]. If $\sigma$ is non-exact, $d_{1}(g, \sigma)$ can be infinite; examples with infinite $d_{1}(g, \sigma)$ are non-exact closed 2-forms $\sigma$ on tori, see [18], [52].

3. One cannot expect that $\mathcal{P}^{\circ}\left(E_{r}\right) \neq \emptyset$ for almost all $r>0$ in general. Indeed, let $M$ be a closed oriented surface of genus 2, and let $g$ and $\sigma$ either be a Riemannian metric of constant curvature -1 and its area form or the Riemannian metric and the exact 2-form constructed in [48]. Then $\mathcal{P}^{\circ}\left(E_{r}\right)=\emptyset$ for all $r \geq \frac{1}{2}$, see [14, Example 3.7] and [48].

4. Assume that $M$ is neither a 2 -sphere nor an orientable surface of genus $\geq 2$. If $\sigma$ is non-exact, then none of the hypersurfaces $E_{r}$ in $\left(T^{*} M, \omega_{\sigma}\right)$ is of contact type, see e.g. [52]. Therefore, Corollary 3.6 does not follow from existence results of closed characteristics on contact type hypersurfaces.

\section{References}

[1] V. Arnol'd, On some problems in symplectic topology. In Topology and geometryRohlin Seminar, Lecture Notes in Math. 1346, Springer-Verlag, Berlin 1988, 1-5. Zbl 0655.58002 MR 0970068 
[2] M. Audin, F. Lalonde and L. Polterovich, Symplectic rigidity: Lagrangian submanifolds. In Holomorphic curves in symplectic geometry, Progr. Math. 117, Birkhäuser, Basel 1994, 271-321. MR 1274934

[3] M. Bialy and L. Polterovich, Invariant tori and symplectic topology. In Sină̌s Moscow Seminar on Dynamical Systems, Amer. Math. Soc. Transl. Ser. 2 171, Amer. Math. Soc., Providence, RI, 1996, 23-33. Zbl 0847.58022 MR 1359090

[4] W. Chen, Pseudo-holomorphic curves and the Weinstein conjecture. Comm. Anal. Geom. 8 (2000), 115-131. Zbl 0978.53135 MR 1730894

[5] K. Cieliebak, V. Ginzburg and E. Kerman, Symplectic homology and periodic orbits near symplectic submanifolds. Comment. Math. Helv. 79 (2004), 554-581. Zbl 02113905 MR 2081726

[6] G. Contreras, The Palais-Smale condition for contact type energy levels for convex lagrangian systems. math.DS/0304238.

[7] G. Contreras, R. Iturriaga, G. P. Paternain and M. Paternain, The Palais-Smale condition and Mañé's critical values. Ann. Henri Poincaré 1 (2000), 655-684. Zbl 0986.58005 MR 1785184

[8] G. Contreras, L. Macarini and G. P. Paternain, Periodic orbits for exact magnetic flows on surfaces. Internat. Math. Res. Notices 2004 (8), 361-387. MR 2036336

[9] M. Entov, K-area, Hofer metric and geometry of conjugacy classes in Lie groups. Invent. Math. 146 (2001), 93-141. Zbl 1039.53099 MR 1859019

[10] A. Floer, H. Hofer and C. Viterbo, The Weinstein conjecture in $P \times \mathbb{C}^{l}$. Math. Z. 203 (1990), 469-482. Zbl 0666.58019 MR 1038712

[11] U. Frauenfelder, V. Ginzburg and F. Schlenk, Energy capacity inequalities via an action selector. In Geometry, Spectral Theory, Groups, and Dynamics (M. Entov, Y. Pinchover, M. Sageev, eds.), Contemp. Math. 387, Amer. Math. Soc., Providence, R.I., 2005, 129-152.

[12] U. Frauenfelder and F. Schlenk, Hamiltonian dynamics on convex symplectic manifolds. math.SG/0303282.

[13] V. Ginzburg, New generalizations of Poincaré's geometric theorem. Funct. Anal. Appl. 21 (1987), 100-106. Zbl 0656.58027 MR 0902290

[14] V. Ginzburg, On closed trajectories of a charge in a magnetic field. An application of symplectic geometry. In Contact and symplectic geometry (Cambridge, 1994), Publ. Newton Inst. 8, Cambridge University Press, Cambridge 1996, 131-148. Zbl 0873.58034 MR 1432462

[15] V. Ginzburg, Hamiltonian dynamical systems without periodic orbits. In Northern California Symplectic Geometry Seminar, Amer. Math. Soc. Transl. Ser. 2 196, Amer. Math. Soc., Providence, RI, 1999, 35-48. Zbl 0958.37044 MR 1736212

[16] V. Ginzburg, The Weinstein conjecture and theorems of nearby and almost existence. In The breadth of symplectic and Poisson geometry, Progr. Math. 232, Birkhäuser Boston, Boston, MA, 2005, 139-172. MR 2103006

[17] V. Ginzburg and B. Gürel, Relative Hofer-Zehnder capacity and periodic orbits in twisted cotangent bundles. Duke Math. J. 123 (2004), 1-47. Zbl 02114444 MR 2060021 
Vol. 81 (2006)

[18] V. Ginzburg and E. Kerman, Periodic orbits in magnetic fields in dimensions greater than two. In Geometry and topology in dynamics (Winston-Salem, NC, 1998/San Antonio, TX, 1999), Contemp. Math. 246, Amer. Math. Soc., Providence, RI, 1999, 113-121. Zbl 0948.37045 MR 1732375

[19] V. Ginzburg and E. Kerman, Periodic orbits of Hamiltonian flows near symplectic extrema. Pacific J. Math. 206 (2002), 69-91. Zbl 1055.37065 MR 1924819

[20] M. Gromov, Pseudo-holomorphic curves in symplectic manifolds. Invent. Math. 82 (1985), 307-347. Zbl 0592.53025 MR 0809718

[21] H. Hofer, On the topological properties of symplectic maps. Proc. Roy. Soc. Edinburgh Sect. A 115 (1990), 25-38. Zbl 0713.58004 MR 1059642

[22] H. Hofer, Estimates for the energy of a symplectic map. Comment. Math. Helv. 68 (1993), 48-72. Zbl 0787.58017 MR 1201201

[23] H. Hofer and D. Salamon, Floer homology and Novikov rings. In The Floer memorial volume, Progr. Math. 133, Birkhäuser-Verlag, Basel 1995, 483-524. Zbl 0842.58029 MR 1362838

[24] H. Hofer and C. Viterbo, The Weinstein conjecture in cotangent bundles and related results. Ann. Scuola Norm. Sup. Pisa Cl. Sci. (4) 15(1988), 411-445.Zbl 0697.58044 MR 1015801

[25] H. Hofer and C. Viterbo, The Weinstein conjecture in the presence of holomorphic spheres. Comm. Pure Appl. Math. 45 (1992), 583-622. Zbl 0773.58021 MR 1162367

[26] H. Hofer and E. Zehnder, Periodic solutions on hypersurfaces and a result by C. Viterbo. Invent. Math. 90 (1987), 1-9. Zbl 0631.58022 MR 0906578

[27] H. Hofer and E. Zehnder, A new capacity for symplectic manifolds. In Analysis, et cetera, Academic Press, Boston, MA, 1990, 405-427. Zbl 0702.58021 MR 1039354

[28] H. Hofer and E. Zehnder, Symplectic Invariants and Hamiltonian Dynamics. Birkhäuser, Basel 1994. Zbl 0805.58003 MR 1306732

[29] M.-Y. Jiang, Hofer-Zehnder symplectic capacity for two-dimensional manifolds. Proc. Roy. Soc. Edinburgh Sect. A 123 (1993), 945-950. Zbl 0796.53035 MR 1249696

[30] E. Kerman, Periodic orbits of Hamiltonian flows near symplectic critical submanifolds. Internat. Math. Res. Notices 1999 (17), 953-969. Zbl 0958.37041 MR 1717637

[31] V. V. Kozlov, Calculus of variations in the large and classical mechanics. Russian Math. Surveys 40 (2) (1985), 37-71. Zbl 0579.70020 MR 0786086

[32] F. Lalonde and D. McDuff, The geometry of symplectic energy. Ann. of Math. (2) 141 (1995), 349-371. Zbl 0829.53025 MR 1324138

[33] F. Lalonde and D. McDuff, Hofer's $L^{\infty}$-geometry: energy and stability of Hamiltonian flows, part I. Invent. Math. 122 (1995), 1-33. Zbl 0844.58020 MR 1354953

[34] F. Lalonde and D. McDuff, Hofer's $L^{\infty}$-geometry: energy and stability of Hamiltonian flows, part II. Invent. Math. 122 (1995), 35-69. Zbl 0844.58021 MR 1354953

[35] F. Laudenbach, Homotopie régulière inactive et engouffrement symplectique. Ann. Inst. Fourier 36 (1986), 93-111. Zbl 0576.57027 MR 0850746

[36] F. Laudenbach and J.-C. Sikorav, Hamiltonian disjunction and limits of Lagrangian submanifolds. Internat. Math. Res. Notices 1994 (4), 161-168. Zbl 0812.53031 MR 126611 
[37] G. Liu and G. Tian, Weinstein conjecture and GW-invariants. Commun. Contemp. Math. 2 (2000), 405-459. Zbl 1008.53071 MR 1806943

[38] G. Lu, The Weinstein conjecture on some symplectic manifolds containing the holomorphic spheres. Kуиshu J. Math. 52 (1998), 331-351 and 54 (2000) 181-182. Zbl 0987.53033 MR 1762803

[39] G. Lu, The Weinstein conjecture in the uniruled manifolds. Math. Res. Lett. 7 (2000), 383-387. Zbl 0983.53062 MR 1783615

[40] R.-Y. Ma, Symplectic capacity and the Weinstein conjecture in certain cotangent bundles and Stein manifolds. Nonlinear Differential Equations Appl. 2 (1995), 341-356. Zbl 0874.53029 MR 1343398

[41] L. Macarini, Hofer-Zehnder capacity and Hamiltonian circle actions. Commun. Contemp. Math. 6 (2004), 913-945. Zbl 02165914 MR 2112475

[42] L. Macarini and F. Schlenk, A refinement of the Hofer-Zehnder theorem on the existence of closed trajectories near a hypersurface. Bull. London Math. Soc. 37 (2005), 297-300. Zbl 02164584 MR 2119029

[43] D. Mc Duff, Symplectic manifolds with contact type boundaries. Invent. Math. 103 (1991), 651-671. Zbl 0719.53015 MR 1091622

[44] D. Mc Duff and J. Slimowitz, Hofer-Zehnder capacity and length minimizing Hamiltonian paths. Geom. Topol. 5 (2001), 799-830. Zbl 1002.57056 MR 1871405

[45] S. P. Novikov, The Hamiltonian formalism and a many-valued analogue of Morse theory. Russian Math. Surveys 37 (5) (1982), 1-56. Zbl 0571.58011 MR 0676612

[46] A. Oancea, The Kunneth formula in Floer homology for manifolds with contact type boundary. math.SG/0403376.

[47] G. Paternain, Magnetic Rigidity of Horocycle flows. math.DS/0409528.

[48] G. Paternain and M. Paternain, Critical values of autonomous Lagrangian systems. Comment. Math. Helv. 72 (1997), 481-499. Zbl 0921.58017 MR 1476061

[49] L. Polterovich, An obstacle to non-Lagrangian intersections. In The Floer memorial volume, Progr. Math. 133, Birkhäuser, Basel 1995, 575-586. Zbl 0847.58038 MR 1362842

[50] L. Polterovich, Geometry on the group of Hamiltonian diffeomorphisms. Proceedings of the International Congress of Mathematicians, Vol. II (Berlin, 1998). Doc. Math. 1998, Extra Vol. II, 401-410. Zbl 0909.58004 MR 1648090

[51] L. Polterovich, The geometry of the group of symplectic diffeomorphisms. Lectures in Mathematics ETH Zürich, Birkhäuser Verlag, Basel 2001. Zbl 01584173 MR 1826128

[52] F. Schlenk, Applications of Hofer's geometry to Hamiltonian dynamics. http://www.math. uni-leipzig.de/ schlenk/Maths/Papers/applications.pdf

[53] M. Schwarz, On the action spectrum for closed symplectically aspherical manifolds. $\mathrm{Pa}$ cific J. Math. 193 (2000), 419-461. Zbl 1023.57020 MR 1755825

[54] P. Seidel, $\pi_{1}$ of symplectic automorphism groups and invertibles in quantum homology rings. Geom. Funct. Anal. 7 (1997), 1046-1095. Zbl 0928.53042 MR 1487754

[55] J.-C. Sikorav, Systèmes Hamiltoniens et topologie symplectique. Dipartimento di Matematica dell' Università di Pisa, 1990, ETS EDITRICE PISA. 
[56] J.-C. Sikorav, Some properties of holomorphic curves in almost complex manifolds. In Holomorphic curves in symplectic geometry, Progr. Math. 117, Birkhäuser, Basel 1994, 165-189. MR 1274929

[57] C. Viterbo, A proof of Weinstein's conjecture in $\mathbb{R}^{2 n}$. Ann. Inst. H. Poincaré Anal. Non Linéaire 4 (1987), 337-356. Zbl 0631.58013 MR 0917741

[58] C. Viterbo, Exact Lagrange submanifolds, periodic orbits and the cohomology of free loop spaces. J. Differential Geom. 47 (1997), 420-468. Zbl 0946.37017 MR 1617648

[59] C. Viterbo, Functors and computations in Floer homology with applications. I. Geom. Funct. Anal. 9 (1999), 985-1033. Zbl 0954.57015 MR 1726235

[60] A. Weinstein, On the hypotheses of Rabinowitz' periodic orbit theorems. J. Differential Equations 33 (1979), 353-358. Zbl 0388.58020 MR 0543704

[61] E. Zehnder, Remarks on periodic solutions on hypersurfaces. Periodic solutions of Hamiltonian systems and related topics (Il Ciocco, 1986), NATO Adv. Sci. Inst. Ser. C Math. Phys. Sci. 209, Reidel, Dordrecht 1987, 267-279. Zbl 0639.58011 MR 0920629

Received May 27, 2003; revised March 2, 2005

Felix Schlenk, Mathematisches Institut, Universität Leipzig, 04109 Leipzig, Germany

E-mail: schlenk@math.uni-leipzig.de 